EPJ Web of Conferences 66, 01008 (2014)

DOI: $10.1051 /$ epjconf/ 20146601008

(C) Owned by the authors, published by EDP Sciences, 2014

\title{
Cluster formation, breaking, and excitation in light nuclei
}

\author{
Y. Kanada-En'yo ${ }^{1}$, T. Suhara ${ }^{2}$, and F. Kobayashi ${ }^{1}$ \\ ${ }^{1}$ Department of Physics, Kyoto University, Kyoto 606-8502, Japan \\ ${ }^{2}$ Matsue College of Technology, Matsue 690-8518, Japan
}

\begin{abstract}
In this paper, we discuss cluster phenomena in light nuclei based on calculations using the antisymmetrized molecular dynamics (AMD) method. Cluster structures in $\mathrm{C}, \mathrm{B}$, and $\mathrm{Be}$ were studied systematically, and their cluster formation and excitation are discussed. Cluster gas states and their band members are suggested for the excited states of ${ }^{12} \mathrm{C}$ and ${ }^{11} \mathrm{~B}$. The ground state cluster correlation and excited cluster gas state are discussed from the point of view of symmetry breaking and restoration.
\end{abstract}

\section{Introduction}

One of the important and well-known features of nuclear systems is the independent particle feature in a mean field. On the other hand, the correlation between nucleons is rather strong because of the attractive nuclear force. A typical spatial correlation is a cluster which is a subunit of many nucleons with strong spatial correlation. Because of the saturation property a nucleus can easily break into many parts with a small amount of energy. As a result, cluster and mean-field states coexist in lowenergy spectra. The coexistence of these two kinds of structure, cluster and mean field features, is the origin of rich phenomena in ground and excited states.

${ }^{12} \mathrm{C}$ is a typical example where cluster and mean-field features coexist. The ground state of ${ }^{12} \mathrm{C}$ is a compact shell-model state with normal density, and it can be described as a nuclear liquid. With energy around $100 \mathrm{MeV}$, all twelve nucleons of ${ }^{12} \mathrm{C}$ can break up, and the system evolves to a nucleon gas state. In the energy region around $10 \mathrm{MeV}$, much below the nucleon gas state, three $\alpha$ clusters develop in excited states of ${ }^{12} \mathrm{C}$. The energy for the $3 \alpha$ cluster excitation is much smaller the nucleon gas state, implying the coexistence of cluster and mean-field states in low-energy spectra. Moreover, the cluster feature contributes to the ground state structure of ${ }^{12} \mathrm{C}$ as a ground state many-body correlation. If there is no correlation between nucleons, they all behave as independent particles in a spherical mean field. In such an uncorrelated state, there exists a clear Fermi surface and shell structure. In reality, however, when the residual interaction is switched on, correlations occur between nucleons due to the spatial correlation between four nucleons at the Fermi surface, and $\alpha$ clusters are formed at the nuclear surface. In such the correlated state, clusters are largely overlapping with each other to form a compact triangle state of the $3 \alpha$ structure. Once $\alpha$ clusters are formed in the system, three $\alpha$ clusters develop spatially with a small amount of energy because the excitation of the inter-cluster motion can be easily activated. In the spatially developed $3 \alpha$ state, weakly interacting $\alpha$ clusters are moving in a dilute density. There, the mean field description breaks down, because such a state comprising weakly interacting clusters is described by a linear combination of many configurations (many Slater determinants). In realistic ${ }^{12} \mathrm{C}$ systems, the ground state is somewhere between 
the uncorrelated and correlated states of the compact triangle state having the $3 \alpha$ core structure, while the spatially developed $3 \alpha$ cluster structures appear in excited states.

In this paper, we focus on the cluster correlation, particularly the four-body spatial correlation between spin-up and spin-down protons and neutrons (" $\alpha$ correlation") with the relative $S$-wave motion between them. The cluster correlation is characterized by two types of features. One is the local concentration (localization) of single-particle wave functions. It is nothing but the cluster formation. The other is the inter-cluster motion, which is approximately decoupled from the nucleon motions inside each cluster, and often shows the delocalization of the cluster position. The compact triangle state of $3 \alpha$ in ${ }^{12} \mathrm{C}$ corresponds to the former (the cluster formation), and the spatially developed $3 \alpha$ cluster structures in excited states of ${ }^{12} \mathrm{C}$ corresponds to the latter. Cluster structures can contain one or both of these features. The model space of the mean-field methods such as the Hartree-Fock model contains some cluster states with cluster formation as well as uncorrelated states. However, it is difficult for the mean-field techniques to express the inter-cluster motion, because it is described by incorporating the higher correlation beyond the mean-field picture.

Cluster structures are well known in light stable nuclei, and have also been suggested to occur in heavier nuclei. The extensive research in physics of unstable nuclei over the years has revealed that cluster structures also appear in unstable nuclei. This indicates that the cluster aspects are one of the essential features in a wide region of the chart of nuclides. In cluster physics, many theoretical approaches have been developed to describe cluster phenomena. Microscopic studies with cluster models such as the resonating group method started in the 1960s, mainly for very light nuclei, for instance, ${ }^{8} \mathrm{Be}$ with the $2 \alpha$-cluster model. These studies were extended to stable nuclei in the $p$-shell and light $s d$-shell region, in the 1970s [1-3]. From the 1990s, with the progress in physics of unstable nuclei, new theoretical approaches with no (or little) a priori cluster assumptions have been developed. These models are applicable to general unstable nuclei over a wide region of the chart of nuclides, and they have been used to investigate cluster structures in unstable nuclei.

We should stress on the recent remarkable development of $a b$ initio calculations. In these years, $a b$ initio calculations became available for light nuclei. One of the important results concerning cluster physics is the formation of two $\alpha$ clusters in ${ }^{8} \mathrm{Be}$ clearly shown by the eight-body ab initio calculation (Variational Monte Carlo method) using realistic nuclear forces[4]. Ab initio calculations have been developed for $A \geq 10$ nuclei and for the scattering of light nuclei. However, the application to heavier systems and excited cluster states is still limited. Here we should remind the reader that the $2 \alpha$ cluster structure in ${ }^{8} \mathrm{Be}$ was successfully described by the cluster models around 40 years ago; thus, model calculations are often useful to clarify the characteristics of various nuclei in an efficient way.

Our aim is to theoretically investigate various cluster phenomena to achieve a systematic understanding of nuclear systems. In this paper, we discuss cluster phenomena in nuclear systems based on calculations using the antisymmetrized molecular dynamics (AMD) method. The AMD model [5-7] can describe features of both cluster and mean field aspects in general nuclei. In particular, shell-model structures and the cluster formation in low-lying states and developed cluster structures in excited states can be described within the AMD framework without assuming the existence of any clusters.

This paper is organized as follows. In the following section, the formulation of the AMD model is explained. In section 3, cluster structures in C, B, and Be isotopes are discussed. In section 4, from the systematics of cluster structures of these isotopes, the formation, breaking, and excitation of clusters are considered. 
INPC 2013

\section{Formulation of AMD}

The AMD model,like many quantum-mechanical methods, is on the energy variation using effective nuclear forces,

In the AMD framework, an $A$-nucleon wave function is given by a Slater determinant of Gaussian wave packets,

$$
\Phi_{\mathrm{AMD}}(\mathbf{Z})=\frac{1}{\sqrt{A !}} \mathcal{A}\left\{\varphi_{1}, \varphi_{2}, \ldots, \varphi_{A}\right\},
$$

where the $i$ th single-particle wave function is written as follows.

$$
\begin{aligned}
\varphi_{i} & =\phi_{\mathbf{X}_{i}} \chi_{i} \tau_{i}, \\
\phi_{\mathbf{X}_{i}}\left(\mathbf{r}_{j}\right) & \propto \exp \left\{-v\left(\mathbf{r}_{j}-\frac{\mathbf{X}_{i}}{\sqrt{v}}\right)^{2}\right\}, \\
\chi_{i} & =\left(\frac{1}{2}+\xi_{i}\right) \chi_{\uparrow}+\left(\frac{1}{2}-\xi_{i}\right) \chi_{\downarrow} .
\end{aligned}
$$

Here $\phi_{\mathbf{X}_{i}}$ and $\chi_{i}$ are spatial and spin functions, respectively, and $\tau_{i}$ is the isospin function which is fixed as up (proton) or down (neutron). We take an optimized value of the width parameter $v$ for each nucleus. Accordingly, an AMD wave function is expressed by a set of variational parameters (determined by the energy variation), $\mathbf{Z} \equiv\left\{\mathbf{X}_{1}, \mathbf{X}_{2}, \cdots, \mathbf{X}_{A}, \xi_{1}, \xi_{2}, \cdots, \xi_{A}\right\}$, which indicate Gaussian centers and spin orientations for all nucleons. The AMD wave function is similar to that used in fermionic molecular dynamics (FMD) [8, 9], which has more generalized wave functions and is often applied in the unitary correlated operator method [10].

To study nuclear structure, the energy variation, and the total-angular-momentum and parity projections are performed in the AMD model space. In the simple version of AMD, the variation is performed after the parity projection but before the total-angular-momentum projection (VBP: variation before projection). For the study of excited states, the variation after spin-parity projection in the AMD (AMD+VAP) is performed along with the superposition of many AMD configurations (the multi-configuration (MC) mixing). For ${ }^{14} \mathrm{C}$ and ${ }^{11} \mathrm{~B}$, the $\mathrm{AMD}+$ generator coordinate method (AMD+GCM) method with the $\beta-\gamma$ constraint [11] is applied. Further details of the present calculations are described in Refs. [12-16]. A general formulation of the AMD method is explained in Refs. [6, 7].

In the AMD wave function, all single nucleons are treated independently with localized Gaussians. Although no clusters are assumed a priori, multi-cluster structures can be described by grouping the positions of single-nucleon Gaussian wave packets. On the other hand, if all Gaussian centers gather around a certain position, the AMD wave function becomes equivalent to a harmonic oscillator shellmodel wave function around this position because of the antisymmetrization effect. Thus, the AMD model space can describe cluster formation and the independent-particle features with the assembling and disassembling of Gaussian wave packets. If a system favors a specific cluster channel, such a cluster structure will be automatically obtained in the energy variation. Moreover, because of the projections of the total-angular-momentum and parity projections and MC mixing, the final wave functions contain higher correlations that are not expressed by a Slater determinant. Thus, the AMD methods are useful to study cluster correlation. Note that, for ${ }^{12} \mathrm{C}$, an uncorrelated spherical state is obtained after the energy variation in the model space of a single Slater determinant without the projections, although the model space contains the triangle $3 \alpha$ configuration of ${ }^{12} \mathrm{C}$ which is a part of the cluster correlation (cluster formation). This is the same as many mean-field calculations for ${ }^{12} \mathrm{C}$. The importance of the projections and MC mixing in cluster correlation is explained in Ref.[7].

The effective nuclear interactions used in the present calculations are the Volkov and MV1 forces $[17,18]$ with the spin-orbit force supplemented by the Coulomb force. 


\section{Cluster structures in $\mathrm{C}, \mathrm{B}$, and $\mathrm{Be}$ isotopes}

In ${ }^{12} \mathrm{C}$, we see a variety of cluster structures comprising $3 \alpha$ clusters. As mentioned before, the ground state of ${ }^{12} \mathrm{C}$ is considered to be the compact triangle state of the $3 \alpha$ cluster structure with a mixing of the $p_{3 / 2}$-shell closed configuration, while $3 \alpha$ cluster structures develop in excited states. For instance, the $0_{2}^{+}$state is considered to be a dilute cluster gas state of three $\alpha$ s where $\alpha$ clusters are weakly interacting like a gas as proposed by Tohsaki et al. and other groups [19,20]. Because of the bosonic behavior of $\alpha$ particles in such a dilute gas state, this state is often discussed in relation with BoseEinstein condensation, the $\alpha$ condensation, in a dilute nuclear matter. Searching for cluster gas states in other nuclei has been performed in theoretical and experimental works [21].

The AMD calculations of ${ }^{12} \mathrm{C}$ and ${ }^{11} \mathrm{~B}$ were performed, and they suggested a similar cluster gas state of $2 \alpha \mathrm{s}$ and a triton in the $3 / 2_{3}^{-}$state of ${ }^{11} \mathrm{~B}[14]$. The calculated results for the $3 / 2_{3}^{-}$state of ${ }^{11} \mathrm{~B}$ agree with the experimentally measured strong monopole transition and weak M1 transition for the $3 / 2^{-}$state at $8.56 \mathrm{MeV}$ [22]. he $3 / 2_{3}^{-}$state of ${ }^{11} \mathrm{~B}$ is characterized by the spatially developed three-center cluster structure with no geometric configuration, namely, it is expressed by a linear combination of various configurations of cluster positions. These features are quite similar to those of ${ }^{12} \mathrm{C}\left(0_{2}^{+}\right)$; therefore, it is a candidate for the $2 \alpha+t$ cluster gas state.

It is interesting to study the rotation of these cluster gas states. Very recently, new excited states in ${ }^{12} \mathrm{C}$ and ${ }^{11} \mathrm{~B}$ were experimentally observed. In ${ }^{12} \mathrm{C}$, the $2_{2}^{+}$state at $9.84(0.06) \mathrm{MeV}$ and the $4_{1}^{+}$state at $13.3 \mathrm{MeV}$ were reported by Itoh et al. [23] and Freer et al. [24]. For ${ }^{11} \mathrm{~B}$, the $9 / 2^{-}$state at $13.03 \mathrm{MeV}$ was observed by Yamaguchi et al. [25]. These excited states are candidates of band members starting from the cluster gas states, ${ }^{12} \mathrm{C}\left(0_{2}^{+}\right)$and ${ }^{11} \mathrm{~B}\left(3 / 2_{3}^{-}\right)$. In Fig. 1, the excitation energies are plotted as a function of spin $J(J+1)$ along with those values obtained from AMD calculations. In the energy spectra, one can see the rotational band feature, the linear dependence on $J(J+1)$ particularly at high spin states, except for the band-head states. Here a question arises; what is the rotation of the cluster gas state. The cluster gas state has no geometric configuration or intrinsic deformation. Naively, a spherical gas state does not have the rotational degree of freedom because of the rotational invariance of the system. In the AMD result, however, it was found that the intrinsic structure changes with the increase of spin. When the spin goes up, the cluster gas states in the band-head states, ${ }^{12} \mathrm{C}\left(0_{2}^{+}\right)$and ${ }^{11} \mathrm{~B}\left(3 / 2_{3}^{-}\right)$, change into geometric structures with intrinsic deformations. Once the intrinsic system is deformed, it can rotate and construct a rotational band. As a result, the spectra deviate from that of the rigid rotor model, but show the change in the moment of inertia. This is one of the interpretations of these bands.

The present assignment of the band-head state in ${ }^{12} \mathrm{C}$ is based on the rather strong $E 2$ transition strength for $2_{2}^{+} \rightarrow 0_{2}^{+}$obtained by the AMD calculation. However, the AMD result shows further strong $E 2$ transition strength for $2_{2}^{+} \rightarrow 0_{3}^{+}$, which suggests the possible alternative interpretation that the $0_{3}^{+}$state can be the band-head state of the $2_{2}^{+}$state. The intrinsic structure of the $0_{3}^{+}$state is dominated by an open triangle $3 \alpha$ configuration and it is similar to those of the $2_{2}^{+}$and $4^{+}$states.

Another interesting problem is the stability of the linear chain $3 \alpha$ structure in $\mathrm{C}$ isotopes. According to AMD and FMD calculations[12, 13, 26], it was suggested that the straight-line linear chain structure is not stable in ${ }^{12} \mathrm{C}$ but only a chain-like open triangle $3 \alpha$ configuration may appear in the higher $0^{+}$state (the $0_{3}^{+}$state in the present calculation), a few $\mathrm{MeV}$ above the $0_{2}^{+}$state. This suggestion is supported by the recent experimental observation of $0^{+}$states around excitation energy of $10 \mathrm{MeV}$ by Itoh et al. [23].

By adding two neutrons, we expected a linear chain structure in the excited state of ${ }^{14} \mathrm{C}$. The structure of ${ }^{14} \mathrm{C}$ was studied with the $\beta-\gamma$ AMD method.As is expected, it is suggested that the $3 \alpha$ linear-chain structure can be stabilized by additional neutrons in the excited state of ${ }^{14} \mathrm{C}[15]$. As a 
result, a $K^{\pi}=0^{+}$rotational band above the ${ }^{10} \mathrm{Be}+\alpha$ threshold energy is proposed, which is formed from the linear chain structure because of its elongated shape.

Also in neutron-rich Be isotopes, a variety of cluster structures have been suggested in the ground and excited states. Let us consider cluster structures of ${ }^{10} \mathrm{Be}$. In the ground state of ${ }^{10} \mathrm{Be}$, the $2 \alpha$ cluster structure weakens because of the two excess neutrons compared with the $2 \alpha$ cluster structure developed in ${ }^{8} \mathrm{Be}$. In the $0_{2}^{+}$state, two $\alpha$ clusters develop well, and excess neutrons move in the longitudinal molecular orbitals, the so-called $\sigma$ orbitals around $2 \alpha$ [27-29]. In the $0_{2}^{+}$state, two $\alpha$ clusters are bonded by valence neutrons at a certain distance and construct the rotational band (Fig. 1). Recently, ${ }^{6} \mathrm{He}+{ }^{4} \mathrm{He}$ cluster resonance states were proposed above the molecular orbital structure [3032]. In these states, two excess neutrons are localized around one $\alpha$ cluster to form a ${ }^{6} \mathrm{He}$ core, and another $\alpha$ cluster moves around the core.

The ${ }^{6} \mathrm{He}+{ }^{4} \mathrm{He}$ resonance state is regarded as the weak coupling cluster state, which differs from the strong coupling cluster of the molecular orbital structure in the $0_{2}^{+}$state. On the other hand, the molecular orbital structure is a new type of cluster structure different from the traditional cluster in stable nuclei, peculiar to neutron-rich Be isotopes. The idea of molecular orbital structures in neutronrich Be was proposed by Seya et al. and von Oertzen et al. (Ref. [28] and references therein). Let us consider the case of ${ }^{10} \mathrm{Be}$; if there is no correlation between nucleons, all ten nucleons in ${ }^{10} \mathrm{Be}$ move as independent particles in a mean field. If the residual interaction is switched on, two $\alpha$ clusters are formed. Once the $2 \alpha$ core is formed, molecular orbitals are constructed by the linear combination of $p$-orbits around each $\alpha$ cluster and valence neutrons occupy the molecular orbitals around two $\alpha$ s. One is the $\pi$-type orbital that corresponds to the $p$ orbit in the limit of small $\alpha$ - $\alpha$ distance, the neutrons of which attract $2 \alpha$ clusters to gain potential energy. The other is the higher nodal $\sigma$ orbital, which is the key orbital for the developed cluster structure of Be isotopes. The $\sigma$ orbital has two nodes along the $\alpha$ - $\alpha$ direction, and therefore gains kinetic energy with increasing of inter-cluster distance. As a result, the $\sigma$-orbital neutrons repel two $\alpha$ clusters outward. Moreover, in a well-developed cluster state of further neutron-rich Be such as ${ }^{11} \mathrm{Be}$ the inversion of single-particle levels between the $\pi$ and $\sigma$ orbitals occurs. Usually, as in the case of ${ }^{10} \mathrm{Be}$, the $\sigma$ orbital is energetically higher than the $\pi$ orbital. Therefore, the normal $\pi$ configuration is the dominant component of the ground state, while the $\sigma$ configuration appears in the $0_{2}^{+}$state of the developed cluster structure. However, in the case of neutron-rich $\mathrm{Be}$, two $\alpha$ clusters develop further and level inversion occurs. Indeed, in the AMD results of Be isotopes, the breaking of the neutron magic number $N=8$ is proposed also in ${ }^{12} \mathrm{Be}$ and ${ }^{13} \mathrm{Be}$ because of the lowering of the $\sigma$ orbital in the cluster states as well as ${ }^{11} \mathrm{Be}$. The results were consistent with a recent experimental observation of a low-lying abnormal parity state, the $1 / 2^{-}$, in

${ }^{13}$ Be reported by Kondo et al.[33].

\section{Discussion}

In this section, we systematically the cluster structures proposed for $\mathrm{Be}$ and $\mathrm{C}$. Let us consider a $2 \alpha$ system and additional nucleons in the molecular $\pi$ and $\sigma$ orbitals around the $2 \alpha$ core. In ${ }^{10} \mathrm{Be}$, two neutrons occupy the lowest molecular orbital, the $\pi$ orbital, and they attract two $\alpha$ clusters to form a compact ground state of ${ }^{10} \mathrm{Be}$. By adding two more protons to the $\pi$ orbital, the system becomes the ground state of ${ }^{12} \mathrm{C}$. Because of the additional two protons, two $\alpha$ clusters are further weakened and partially broken to form the compact shell-model state in ${ }^{12} \mathrm{C}$. On the other hand, since there are four nucleons in the $\pi$ orbitals, two protons and two neutrons, four-body correlation may occur to form an $\alpha$ cluster at the surface of the $2 \alpha$ system. As a result of the cluster breaking and formation, the ${ }^{12} \mathrm{C}$ ground state becomes the admixture of the shell-model $p_{3 / 2}$-closed configuration and the $3 \alpha$ core structure. By adding two more neutrons in the $\pi$ orbital, the clusters are broken in the ground state of ${ }^{14} \mathrm{C}$ which is the shell-model state. 

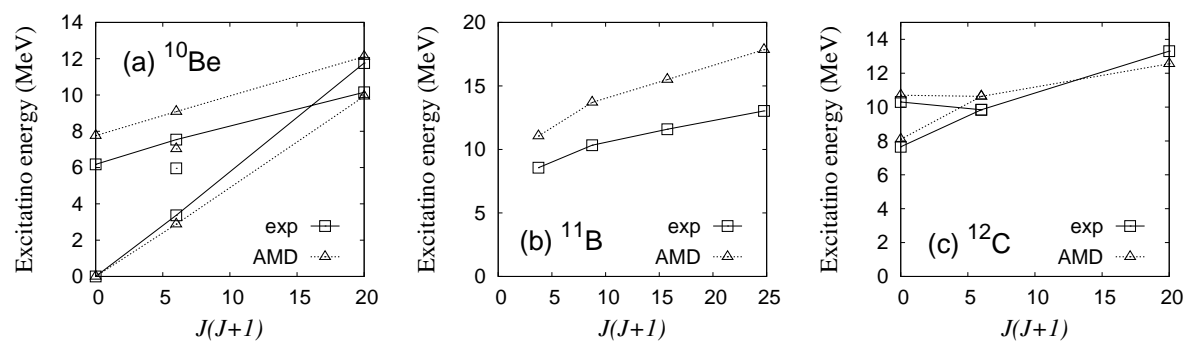

Figure 1. (a) Experimental (exp) and calculated (AMD) excitation energies of $K^{\pi}=0^{+}$-band members of ${ }^{10} \mathrm{Be}$ plotted as functions of the total spin $J(J+1)$. Experimental data are taken from Refs. [34, 35]. The calculated data are those of from the AMD+VAP with the MC mixing from Ref. [36]. (b) and (c) Excitation energies of band members of clusters gas states in ${ }^{11} \mathrm{~B}$ and ${ }^{12} \mathrm{C}$. The band starting from the $J^{\pi}=3 / 2_{3}^{-}$state in ${ }^{11} \mathrm{~B}$ and that from the $0_{2}^{+}$state in ${ }^{12} \mathrm{C}$ are shown. Experimental data are taken from Refs. [23-25, 34]. Calculated values are those of the AMD+GCM from Ref. [16] for ${ }^{11} \mathrm{~B}$ and those of the AMD+VAP with the MC mixing from Ref. [13].

Next we consider the $\sigma$ orbital configurations rather than the $\pi$ orbital configurations. As already mentioned, two neutrons in the $\sigma$ orbital repel $2 \alpha$ clusters outward to form the developed cluster structure, which corresponds to the $0_{2}^{+}$state of ${ }^{10} \mathrm{Be}$. The differences in the occupied molecular orbital are the main origin of the differences in the development of the cluster structures between the $0_{1}^{+}$and $0_{2}^{+}$in ${ }^{10} \mathrm{Be}$. By adding two more protons to the $\sigma$ orbital, the $2 \alpha$-cluster structure develops further, and simultaneously, the four-body correlation occurs between the two protons and two neutrons in the $\sigma$ orbital. In the case of $\sigma$ configuration, the four-body correlation is strong enough to form an almost isolated $\alpha$ cluster. The $\alpha$ cluster then favors to lie at the bent position rather than the straight-line position to release the kinetic energy of the cluster motion. Thus the open triangle configuration of $3 \alpha$ clusters appears in the excited states of ${ }^{12} \mathrm{C}$ as suggested for the $0_{3}^{+}$state by the AMD results. In other words, the axial symmetry, which is seen in the molecular orbital structures of the $0_{2}^{+}$state of ${ }^{10} \mathrm{Be}$, is broken in the $0_{3}^{+}$state of ${ }^{12} \mathrm{C}$ because of the four-body $(\alpha)$ correlation. By adding two more neutrons, the linear chain structure is stabilized again by the excess neutrons in the excited state of ${ }^{14} \mathrm{C}$, and the axial symmetry is restored once more.

Thus, by extending molecular orbital structures and considering the cluster formation of nucleons in the molecular orbitals we can understand those strong-coupling cluster states in $\mathrm{Be}$ and $\mathrm{C}$. For other cluster states such as the cluster gas state in ${ }^{12} \mathrm{C}\left(0_{2}^{+}\right)$and the ${ }^{6} \mathrm{He}+\alpha$ resonance state in ${ }^{10} \mathrm{Be}$, the molecular orbital description is not applicable, because they are weak-coupling cluster states. These cluster states are obtained from the excitation of the inter-cluster motion, i.e., the cluster excitation constructed on the ground state, which already contains the corresponding cluster core.

Finally, we discuss the ground state $\alpha$ correlation and the cluster gas state in ${ }^{12} \mathrm{C}$ from the point of view of symmetry breaking and restoration. As already mentioned, both mean-field and cluster structures coexist in ${ }^{12} \mathrm{C}$. At the limit of no correlation, the state is characterized by the independent single particle motion in a spherical mean field. In contrast, developed cluster states correspond to the condition of strong correlation. Let us consider the intrinsic shape change from the spherical meanfield condition to the cluster condition, i.e., from the limit with no correlation to the limit with strong correlation. In the no correlation limit, the system has a spherical shape with rotational symmetry because of the rotational invariance of the original Hamiltonian. With weak correlation, the structure changes into an oblate shape with axial symmetry. If the cluster cores are formed at the surface because of multi-nucleon correlation, then the intrinsic structure changes into a triangle shape with three $\alpha$-cluster cores, and the axial symmetry is broken. With cluster excitation, the system becomes a 
cluster gas state with low density, and it transforms again to the spherical state. This means that with correlation the rotational symmetry $\mathrm{O}(3)$ is broken into the D3h symmetry of the triangular compact $3 \alpha$ state, and is restored again into $\mathrm{O}(3)$ symmetry in the strong correlation limit in a low density. Notably, the symmetry is broken in the triangle state because of the formation of cluster cores at the nuclear surface; however, with further development of the cluster structure, the symmetry is restored in the cluster gas state.

The breaking and restoration of the (axial) symmetry have an analogy with the phase transition of infinite matter. The rotational symmetry in a finite system can be associated with the translational symmetry in infinite matter. At the limit of no correlation, the spherical state with the rotational symmetry corresponds to the Fermi gas state with translational symmetry. At the strong correlation limit, the spherical cluster gas state corresponds to the alpha condensation state with translational invariance. Moreover, the rotational symmetry is broken in the triangle state having an oscillating periodic density at the surface, which is analogous to the breaking of translational symmetry in the static density wave (DW) phase having oscillating inhomogeneous density. What we found is that one can interpret the triangle state with cluster core correlation as the DW at the edge of the oblate state; i.e., the ground state cluster correlation in ${ }^{12} \mathrm{C}$ can be understood by the angular DW analogous to the DW phase in infinite matter. In general, the DW is favored in low dimensional systems but not in higher dimensional systems. Therefore, in the realistic 3D nuclear matter, DW is not considered to occur. However, the angular DW in finite nuclei is, in principle, the 1D DW at the edge of the oblate state. This is the reason why the DW can be realized as the ground state correlation, at least in the light nuclear region, as proposed for ${ }^{28} \mathrm{Si}$ [37]. In heavier nuclei, the DW may be destroyed by the spin-orbit force and the BCS phase is considered to be favored rather than the DW phase. Also in the case of a neutron-rich system, the DW may be weakened in general because of the different Fermi surfaces of protons and neutrons. This is consistent with the weaker cluster structure in the ground state of ${ }^{14} \mathrm{C}$ compared to ${ }^{12} \mathrm{C}$.

\section{Summary}

Clusters are an essential feature of nuclear systems. The coexistence of mean-field and cluster features is the origin of rich phenomena in ground and excited states of both stable and unstable nuclei. In this paper, some cluster phenomena in light nuclei were discussed based on the AMD model. The cluster structures in C, B, and Be were described. Finally, the cluster formation and cluster excitation were discussed to understand the origins of cluster structures.

The ground state cluster correlation and excited cluster gas state were discussed from the point of view of symmetry breaking and restoration. It is also interesting to consider the analogy of cluster phenomena with many-body correlations in other quantum systems.

\section{Acknowledgments}

The computational calculations in this work were performed using the supercomputers at YITP and KEK. This work was supported by Grant-in-Aid for Scientific Research from the Japan Society for the Promotion of Science (JSPS). It was also supported by the Grant-in-Aid for the Global COE Program "The Next Generation of Physics, Spun from Universality and Emergence" from the Ministry of Education, Culture, Sports, Science and Technology (MEXT) of Japan.

\section{References}

[1] K. Ikeda et al., Prog. Theor. Phys. Suppl. 52, 1 (1972) 
[2] K. Ikeda et al., Prog. Theor. Phys. Suppl. 62, 1 (1977)

[3] K. Ikeda et al., Prog. Theor. Phys. Suppl. 68, 1 (1980)

[4] R. B. Wiringa, S. C. Pieper, J. Carlson and V. R. Pandharipande, Phys. Rev. C 62, 014001 (2000)

[5] Y. Kanada-Enyo, H. Horiuchi and A. Ono, Phys. Rev. C 52, 628 (1995); Y. Kanada-Enyo and H. Horiuchi, Phys. Rev. C 52, 647 (1995)

[6] Y. Kanada-En'yo and H. Horiuchi, Prog. Theor. Phys. Suppl. 142, 205 (2001); Y. Kanada-En'yo M. Kimura and H. Horiuchi, C. R. Physique 4, 497 (2003)

[7] Y. Kanada-En'yo, M. Kimura and A. Ono, PTEP 2012, 01 A202 (2012)

[8] H. Feldmeier, Nucl. Phys. A 515147 (1990)

[9] H. Feldmeier, K. Bieler and J. Schnack Nucl. Phys. A 586493 (1995)

[10] T. Neff and H. Feldmeier, Nucl. Phys. A 713, 311 (2003)

[11] T. Suhara and Y. Kanada-En'yo, Prog. Theor. Phys. 123, 303 (2010)

[12] Y. Kanada-En'yo, Phys. Rev. Lett. 81, 5291 (1998)

[13] Y. Kanada-En’yo, Prog. Theor. Phys. 117, 655 (2007) [Erratum-ibid. 121, 895 (2009)]

[14] Y. Kanada-En'yo, Phys. Rev. C 75, 024302 (2007)

[15] T. Suhara and Y. Kanada-En'yo, Phys. Rev. C 82, 044301 (2010)

[16] T. Suhara and Y. Kanada-En'yo, Phys. Rev. C 85, 054320 (2012)

[17] A. B. Volkov, Nucl. Phys. 7433 (1965)

[18] T. Ando, K. Ikeda and A. Tohsaki, Prog. Theory. Phys. 64, 1608 (1980)

[19] A. Tohsaki, H. Horiuchi, P. Schuck and G. Ropke, Phys. Rev. Lett. 87, 192501 (2001)

[20] Y. Funaki, A. Tohsaki, H. Horiuchi, P. Schuck and G. Ropke, Phys. Rev. C 67, 051306 (2003)

[21] Y. Funaki, T. Yamada, H. Horiuchi, G. Ropke, P. Schuck and A. Tohsaki, Phys. Rev. Lett. 101, $082502(2008)$

[22] T. Kawabata et al., Phys. Lett. B 646, 6 (2007)

[23] M. Itoh et al., Phys. Rev. C 84, 054308 (2011)

[24] M. Freer et al., Phys. Rev. C 83, 034314 (2011)

[25] H. Yamaguchi et al., Phys. Rev. C 83, 034306 (2011)

[26] M. Chernykh, H. Feldmeier, T. Neff, P. von Neumann-Cosel and A. Richter, Phys. Rev. Lett. 98, 032501 (2007)

[27] M. Seya, M. Kohno, and S. Nagata, Prog. Theor. Phys. 65, 204 (1981)

[28] W. von Oertzen, M. Freer and Y. Kanada-En'yo, Phys. Rep. 43243 (2006)

[29] N. Itagaki and S. Okabe, Phys. Rev. C 61, 044306 (2000)

[30] M. Ito, Phys. Lett. B 636, 293 (2006)

[31] A. N. Kuchera et al., Phys. Rev. C 84, 054615 (2011) [Erratum-ibid. C 85, 069902 (2012)]

[32] F. Kobayashi and Y. Kanada-En'yo, Phys. Rev. C 86, 064303 (2012)

[33] Y. Kondo et al., Phys. Lett. B 690, 245 (2010)

[34] D. R. Tilley et al., Nucl. Phys. A 745155 (2004)

[35] M. Freer et al., Phys. Rev. Lett. 96, 042501 (2006)

[36] Y. Kanada-En'yo, H. Horiuchi and A. Dote, Phys. Rev. C 60, 064304 (1999)

[37] Y. Kanada-En'yo and Y. Hidaka, Phys. Rev. C 84, 014313 (2011) 